\title{
Análisis de Ruido en el Diseño y Construcción de una Cámara Semianecoica para Diagnóstico de Motores de Combustión Interna
}

\author{
Milton O. García ${ }^{(1,2)}$, Carlos G. Ordóñez ${ }^{(1,2)}$ y Antonio J. Torregrosa ${ }^{(3)}$ \\ (1) Ingeniería Mecánica Automotriz, Universidad Politécnica Salesiana, Sede Cuenca, Cuenca -Ecuador. \\ (2) Grupo de Investigación en Ingeniería del Transporte (GIIT), Universidad Politécnica Salesiana, Sede \\ Cuenca, Cuenca - Ecuador (e-mail: mgarciat@ups.edu.ec; gabodtc123@hotmail.com) \\ (3) CMT - Motores Térmicos, Universitat Politècnica de València, Valencia - España \\ (e-mail: atorreg@mot.upv.es)
}

Recibido Ene. 4, 2016; Aceptado Mar. 7, 2016; Versión final Abr. 1, 2016, Publicado Oct. 2016

\begin{abstract}
Resumen
Se presentan los resultados del diseño, implementación y evaluación de una cámara semianecoica que garantice la fiabilidad en la toma de datos para el diagnóstico de sistemas mecánicos. El análisis de ruido es una de las técnicas de mantenimiento predictivo que permite detectar fallos en base al monitoreo de parámetros característicos del buen funcionamiento de un sistema. Para el trabajo, se determinaron las características aislantes y absorbentes de los materiales, así como su disposición dentro de la cámara. La evaluación se realizó mediante el análisis de las señales acústicas emitidas por una fuente (motor de combustión interna Diésel) durante su funcionamiento con y sin fallos provocados. Estas señales fueron adquiridas mediante un sonómetro y analizadas en el dominio de la frecuencia utilizando la transformada rápida de Fourier.
\end{abstract}

Palabras clave: análisis de ruido; mantenimiento predictivo; cámara semianecoica; materiales aislantes; transformada de Fourier

\section{Noise Analysis in the Design and Construction of a Semianechoic Chamber for the Diagnosis of Internal Combustion Engines}

\begin{abstract}
The results of the design, implementation and evaluation of a semi-anechoic chamber to guarantee reliability in data acquisition for diagnosis of mechanical systems are presented. Noise analysis is one of the predictive maintenance techniques that detect faults based on the monitoring of characteristic parameters of proper functioning of a system. To do this, the characteristics of insulating and absorbent material were determined, and their arrangement inside the chamber. The evaluation was performed using the analysis of acoustic signals emitted by a source (internal combustion Diesel engine) under its normal and failure operation. These signals were acquired using a sound level meter and analyzed in the frequency domain using fast Fourier transform.
\end{abstract}




\section{INTRODUCCIÓN}

Una cámara anecoica o también conocida como local de campo libre, consiste en un laboratorio diseñado para minimizar las reflexiones y reverberaciones del sonido, y a la vez eliminar en lo posible los ruidos procedentes del exterior. La combinación de las propiedades de absorción y aislamiento acústico genera que la sala emule las condiciones acústicas que se darían en un campo libre, de esta forma se puede grabar o reproducir un sonido sin ningún tipo de interferencia ni distorsión, por lo que una cámara anecoica sigue siendo la única herramienta confiable para realizar mediciones precisas de sonido. El acondicionamiento de la cámara semianecoica se realizó mediante una minuciosa elección de materiales y elementos acorde a las especificaciones citadas en la norma ISO 3745:2012, siendo el factor más determinante, la frecuencia de corte. Esta frecuencia es aquella donde la cámara muestra un comportamiento totalmente anecoico y nace a partir de los armónicos fundamentales correspondientes a la frecuencia de encendido $1 x$ de un Motor de Combustión Interna. Un adecuado acondicionamiento acústico suprimirá lo máximo posible las reflexiones de las ondas incidentes, por lo que el material de absorción es un elemento de mucha importancia para eliminar ecos y minimizar la reverberación (Rodríguez et al, 2001).

Se han realizado ya varias investigaciones anteriores para el diseño de cámaras anecoicas y semianecoicas, como la llevada a cabo por Piedrahita y Fajardo (2012) donde se realiza un estudio de la pérdida de transmisión sonora (PT) en diversos materiales de uso cotidiano, tales como: icopor, cartón, vidrio, algodón y fique, en función de la frecuencia y barriendo un espectro en bandas de tercio de octava desde los $100 \mathrm{~Hz}$ hasta $5.000 \mathrm{~Hz}$. De la misma forma, en la investigación de Kim et al (2007) se plantea el diseño para la construcción de una cámara anecoica a fin de satisfacer la absorción de ondas electromagnéticas en un ancho de banda de frecuencias de $30 \mathrm{MHz}$ a $20 \mathrm{GHz}$. Por otra parte, Davidson (2014) sigue las mismas especificaciones citadas en la norma ISO 3745:2012 para una cámara anecoica orientada a fuentes omnidireccionales de tamaño reducido (6x9 in) para comparar resultados con estudios realizados en habitaciones sin ningún acondicionamiento acústico previo, así como la determinación de los puntos netamente anecoicos dentro del experimento. Un planteamiento más específico es el que presenta Martyr y Plint (2012) donde resume la normativa y el propósito del uso de células anecoicas en el muestreo de vibraciones y ruido para pruebas de diagnóstico de computadoras automotrices.

Teniendo en cuenta los antecedentes presentados, se desarrolla el presente trabajo, cuyo resultado es la construcción de una cámara semianecoica para la aplicación del análisis de ruido como técnica de mantenimiento predictivo orientado al monitoreo de Motores Diésel. La efectividad de la cámara se puede determinar mediante varias técnicas y/o criterios, de entre las cuales se destaca el trabajo realizado por Schneider (2009) donde su investigación se centra en la predicción numérica mediante el modelado matemático durante la fase de planificación, tomando como variables la geometría del revestimiento acústico y sus condiciones de montaje. Otro criterio para el análisis de la eficiencia de la cámara es el desarrollado en el presente trabajo, cuyo principio se basa en la captación y análisis de señales acústicas emitidas por la fuente (Motor de Combustión). Las señales acústicas captadas pueden ser procesadas mediante varías técnicas, donde destacan el análisis temporal y espectral. El primero implica un análisis de la forma de onda de la señal, resultando un método poco utilizado debido a la complejidad que este representa para señales no estacionarias como la del motor. Por otra parte, el análisis espectral presenta una notable ventaja frente al análisis de la forma de onda en el dominio el tiempo para maquinas alternativas, debido a que la energía contenida en la señal temporal se ve reflejada en el dominio de la frecuencia (Ambardar, 2002), tal y como se indica en la Figura 1.
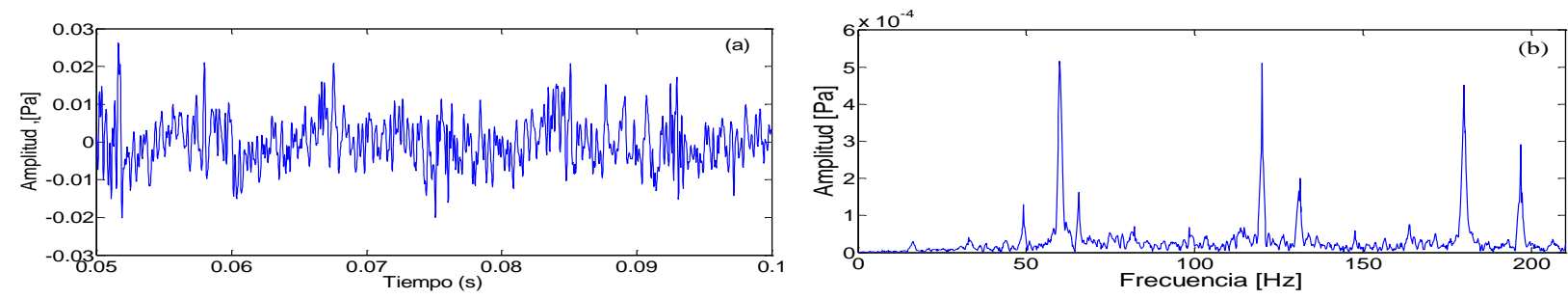

Fig. 1: Análisis de la señal acústica de un motor (a) dominio temporal, (b) dominio frecuencial.

\section{REQUERIMIENTO DE MATERIALES ACÚSTICOS: TIPOS Y PROPIEDADES}

El estudio de los materiales para la construcción de la cámara semianecoica se realizó mediante el Método de Máximos y Mínimos en el Tubo de Kundt (Tubo de Impedancia), el cual permite medir las propiedades acústicas de diferentes materiales por medio de muestras del mismo (probetas), resultando un método altamente confiable, que permite calcular el coeficiente de absorción y pérdida por transmisión del ruido de cada material. 
El método basa su principio en que la energía total que choca en la probeta de prueba se descompone en tres partes, una que se refleja, otra que es absorbida por el material muestreado y la última es transmitida al espacio por detrás de la probeta. Aplicando el principio de conservación de energía se dividen estos fragmentos relacionándose entre sí según la Ecuación 1.

$$
|r|^{2}+\alpha+|t|^{2}=1
$$

Donde $|r|^{2}$ : Coeficiente de Reflexión de Energía, $\alpha$ : Coeficiente de Absorción y $|t|^{2}$ : Coeficiente de Transmisión de Energía.

La ecn. (2) define la relación entre la presión máxima y la presión mínima como $\mu$, logrando medir estas diferentes presiones al resonar la onda en un tubo, formándose de esta manera ondas estacionarias, la presión incidente $P_{i}$ (antinodo) es la máxima presión y la presión reflejada $P_{r}$ (nodo) es la mínima presión dentro del tubo como se indica en la Figura 2 (Möser y Barros, 2009).

$$
\mu=\frac{P_{\min }}{P_{\max }}=\frac{P r}{P i}=\frac{(A-B)}{(A+B)}
$$

El coeficiente de reflexión $r$ es definido por la Ecuación 3:

$$
r=\frac{1-\mu}{1+\mu}
$$

Si la energía que traspasa la muestra es despreciable en comparación con la energía absorbida, el coeficiente de absorción de energía se representa en la Ecuación 4.

$$
\propto=1-r^{2}
$$

El rendimiento del material será mayor cuando más se aproxime el espesor del material a un cuarto de longitud de onda del ruido generado, es decir el máximo espesor se determina por la frecuencia más baja del espectro del ruido (Möser y Barros, 2009).

\section{Materiales aislantes}

El aislamiento acústico evita que los sonidos generados dentro de un recinto se transfiera hacia el exterior y recíprocamente desde este punto hacia el entorno que pretendemos aislar. Este fenómeno se logra impidiendo la propagación de una onda sonora a través del aire, mediante obstáculos reflectores tales como paredes duras y pesadas que reflejen estas ondas. El índice de aislamiento es directamente proporcional a la densidad superficial de la pared o tabique separador, es por ello que una pared mucho más ancha, y por consiguiente más pesada, tendrá un índice de aislamiento mayor que una pared de menor grosor y peso. Experimentalmente por el método de Máximos y Mínimos en el Tubo de Kundt se han obtenido los datos acústicos de diferentes probetas que se detallan a continuación.

a) Yeso Laminado: La obtención de una pérdida de transmisión sonora adecuada se logra mediante la utilización de planchas de yeso laminado, las mismas que están constituidas por un núcleo de roca de yeso y revestidas en sus dos extremos por celulosa. Para aumentar la eficiencia de la pérdida de transmisión acústica, el tabique se rellena con materiales que tengan baja transmisión sonora.

b) Espuma de Polietileno: La Espuma de Polietileno es una material que posee una estructura formada con miles de pequeñas celdas herméticas que forman una barrera impenetrable contra las ondas acústicas.

c) Fibra de Vidrio: La Lana de Vidrio se constituye por filamentos de estructura abierta, ordenada y elástica. Las ondas sonoras que penetran en ella se amortiguan, haciendo que el sonido transmitido al otro lado o reverberado hacia el mismo local, sea menor. De este modo, la transmisión de ruidos: aéreo, de impacto y de sonido reverberado es casi nulo.

La Figura 2, muestra los índices de aislamiento obtenidos en la experimentación de los tres materiales. Una onda acústica se atenúa cada vez que cambie de material, debido a que presenta una reflexión por cada cambio de medio, por lo cual las paredes dobles rellenadas con materiales con baja transmisión de sonido brindan un mayor aislamiento acústico. Una pared compuesta tiene un mayor índice de aislamiento acústico debido al cambio de materiales. Mientras que una pared simple contiene un índice de atenuación constante para una onda acústica. 


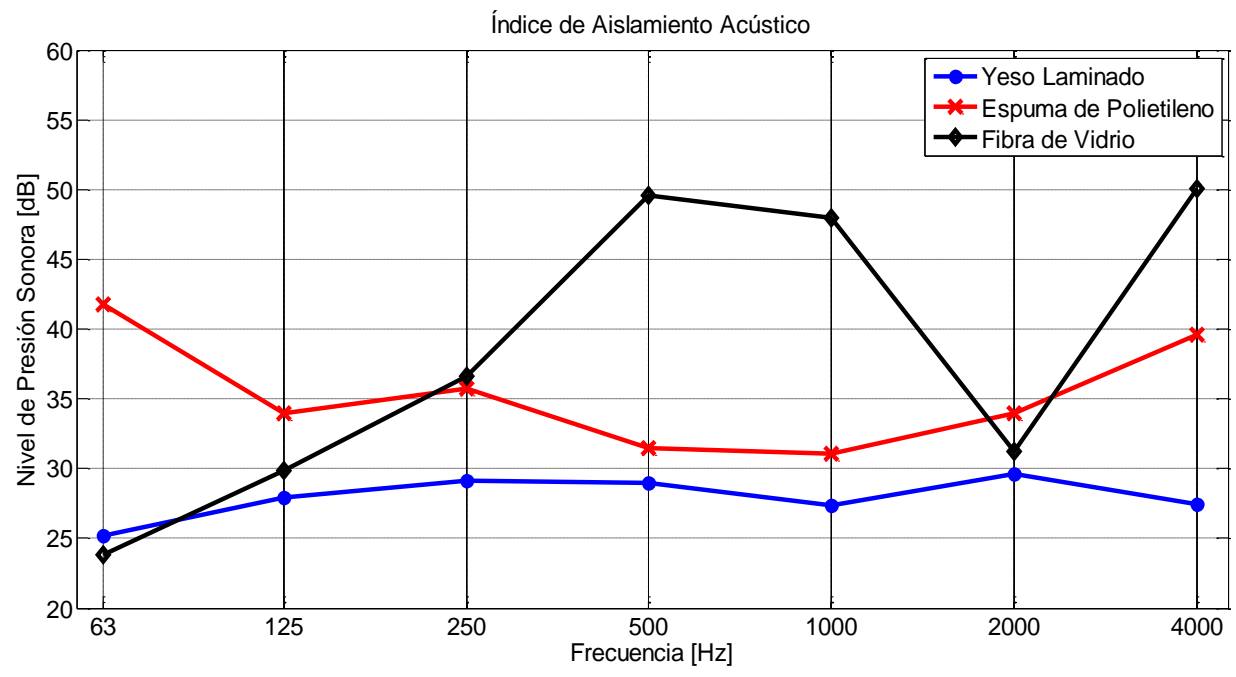

Fig. 2: Pérdida por transmisión sonora de la Fibra de Vidrio - Espesor: 50.8mm.

La configuración de la estructura del aislamiento acústico de la Cámara Semianecoica está distribuida del exterior hacia el interior según lo detallado en la Tabla 1.

Tabla 1: Configuración de la estructura del aislamiento de la Cámara Semianecoica.

\begin{tabular}{|l|c|}
\hline \multicolumn{1}{|c|}{ Material } & Espesor \\
\hline Enlucido Exterior (Mortero) & 0.18 \\
\hline Pared de Bloques de Ladrillo & 0.19 \\
\hline Enlucido Interior (Mortero) & 0.20 \\
\hline Placa de Yeso Laminado & 0.21 \\
\hline Espuma de Polietileno & 0.22 \\
\hline Placas de Fibra de Vidrio & 0.23 \\
\hline Placa de Yeso Laminado & 0.24 \\
\hline
\end{tabular}

\section{Materiales absorbentes}

Experimentalmente se determinó que las superficies de un material aislante reflejan parcialmente el sonido que incide sobre el mismo, la energía restante de la onda incidente debe ser absorbida por una estructura que mecánicamente la transforme en energía cinética y energía calorífica. El rendimiento de esta disipación viene dado por el coeficiente de absorción sonora, el cual depende del tipo, forma y recubrimiento del material, el cual debe ser poroso en toda su densidad. El rendimiento de esta disipación será mayor cuanto más se aproxime el espesor del material a un cuarto de longitud de onda del ruido emitido por la fuente, es decir el máximo espesor se determina por la frecuencia más baja del espectro del ruido.

El análisis acústico en el Tubo de Kundt del Coeficiente de Absorción ( $\alpha$ ), se realizó en dos clases de material, esponja gris y esponja blanca. La primera tiene un menor coeficiente de absorción con respecto a la segunda en bajas frecuencias, debido a que la estructura interna de la esponja blanca presenta mayor porosidad, y como consecuencia, su coeficiente de absorción a bajas frecuencias es mayor. Cuanto más aire estático posea, mayor será el coeficiente de absorción para las frecuencias graves. En la Figura 3 se presenta de forma comparativa el coeficiente de absorción a diferentes frecuencias de los dos materiales.

\section{Diseño y Construcción de la cuña de la Cámara Semianecoica}

Las cuñas de la Cámara Semianecoica se diseñaron en espuma de poliuretano flexible de baja densidad, las espumas poseen diferentes densidades en un rango de 10 a $80 \mathrm{~kg} / \mathrm{m}^{3}$, hechas de polímero ligeramente reticulado con estructura de celda abierta. Este tipo de esponja no contiene barreras entre las células adyacentes lo que permite que el aire fluya a través de la espuma. La Figura 4 representa el esquema de una cuña anecoica con una longitud de un cuarto de longitud de onda, mientras que la Tabla 2 detalla las características técnicas de la cuña usada en la Cámara. 


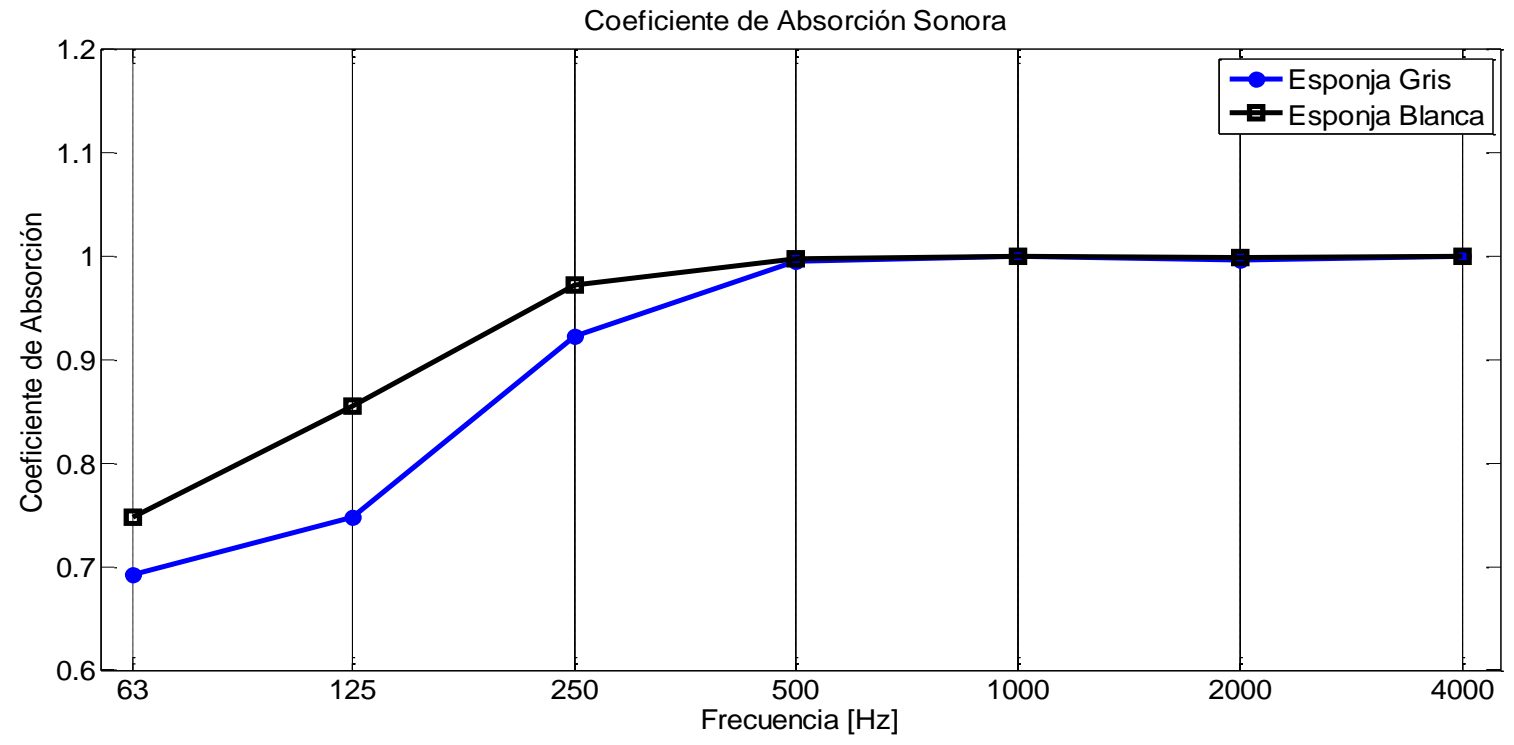

Fig. 3: Coeficiente de absorción sonora $(\alpha)$ de la esponja gris y blanca.

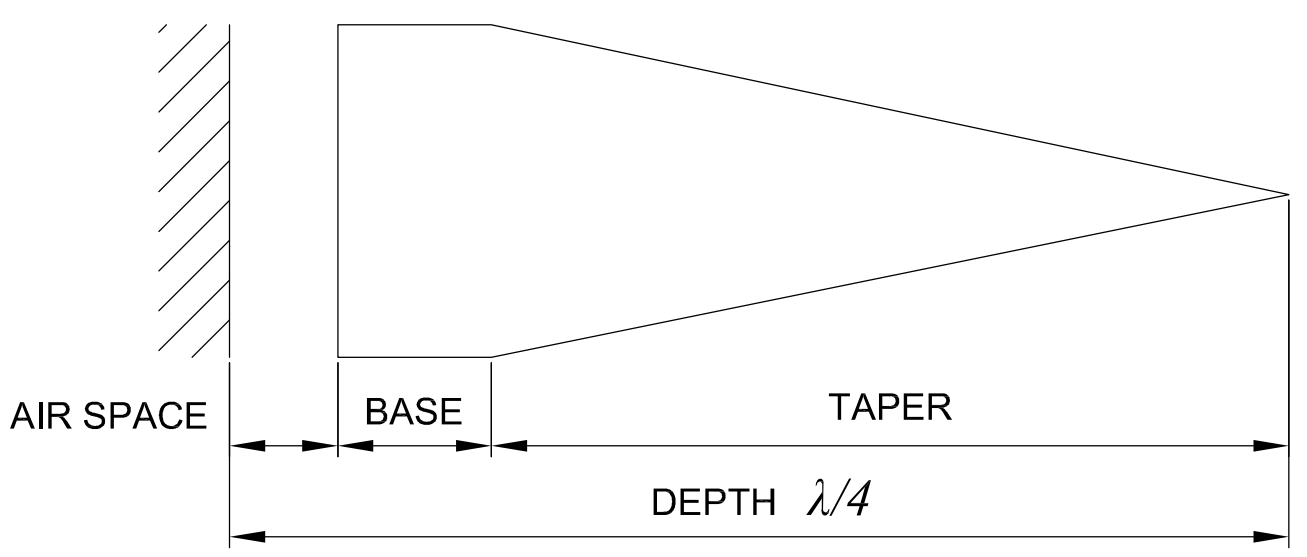

Fig. 4: Absorbente en forma de cuña y longitud de $N / 4$.

Con los absorbentes porosos se logran coeficientes de absorción a incidencia normal superiores al 99\%, a partir de una determinada frecuencia llamada frecuencia de corte. Consiguiendo un aislamiento acústico adecuado del exterior al interior y viceversa, conjuntamente con una absorción anecoica, a continuación en la Figura 5 se visualiza la disposición estructural de los materiales dentro de la Cámara Semianecoica.

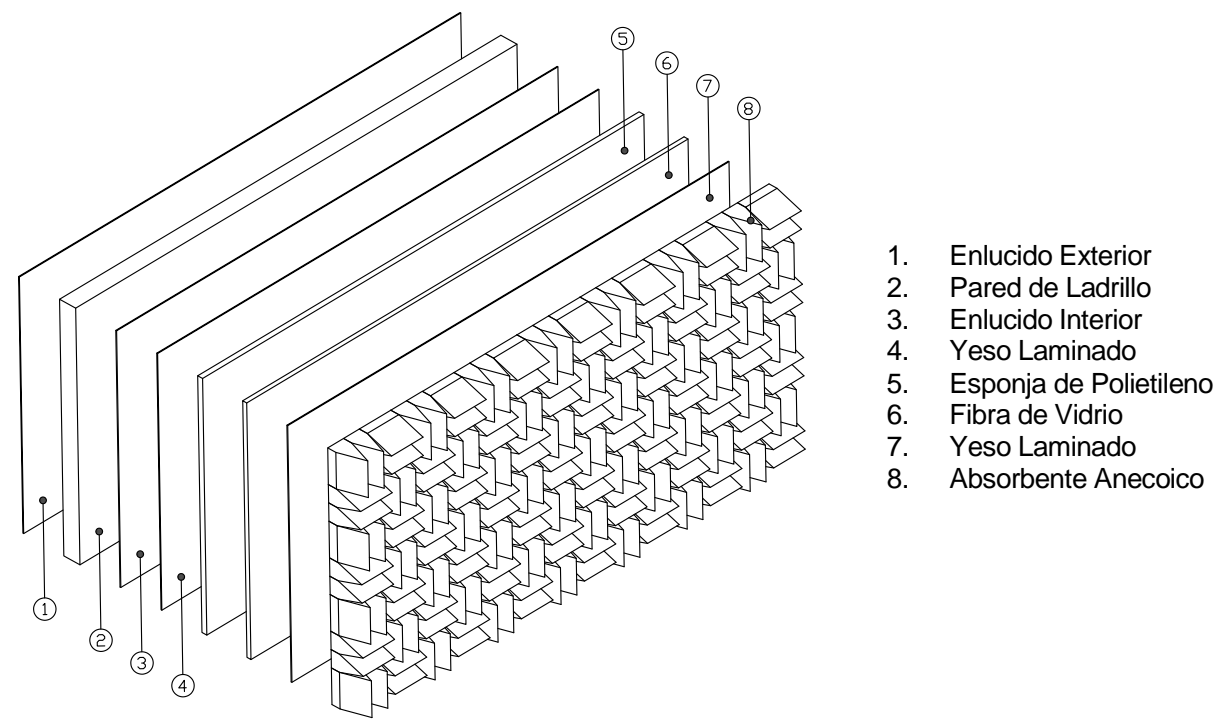

Fig. 5: Configuración de la estructura de la Cámara Semianecoica. 
Tabla 2: Características técnicas de la cuña.

\begin{tabular}{|l|l|}
\hline \multicolumn{1}{|c|}{ Característica } & \multicolumn{1}{c|}{ Descripción } \\
\hline Base & $270 \times 270 \mathrm{~mm}$ \\
\hline Altura & $400 \mathrm{~mm}$ \\
\hline Color & Blanco \\
\hline Densidad & $11,3 \mathrm{~kg} / \mathrm{m}^{3}$ \\
\hline Resistencia al fuego & Media flamabilidad \\
\hline Resistencia a la temperatura & Máx. $150^{\circ} \mathrm{C}$ \\
\hline
\end{tabular}

\section{ANÁLISIS DE LA ACÚSTICA DE RECINTOS: MODOS PROPIOS DE VIBRACIÓN}

Por teoría de vibraciones, se sabe que todo elemento es susceptible a vibración al separarlo de su posición de equilibrio, y al dejarlo libre vibra siempre a unas determinadas formas que corresponden, dentro del entorno acústico, a lo que se denomina Modos Naturales de Vibración. Cada modo lleva asociado una frecuencia característica que se denomina Frecuencia Propia.

Los fundamentos para la determinación de los Modos Propios de Vibración fueron planteados por Strutt (2011), quien define las ecuaciones de los modos propios de vibración en diferentes tipos de condiciones y medios, cuya expresión se define por la Ecuación 5, denominada: La Fórmula de Rayleigh.

$$
f_{x, y, z}=\frac{c}{2} \sqrt{\left(\frac{n_{x}}{L_{x}}\right)^{2}+\left(\frac{n_{y}}{L_{y}}\right)^{2}+\left(\frac{n_{z}}{L_{z}}\right)^{2}}
$$

Donde $c$ : Velocidad del sonido $\left[\mathrm{m} / \mathrm{s}^{2}\right], L_{x}, L_{y}, L_{z}$ : Largo, ancho, alto del recinto; $n_{x}, n_{y}, n_{z}$ : Cualquier valor entero $(0,1,2,3 \ldots . . . n)$.

Según la trayectoria que siguen los frentes de onda, se pueden clasificar los modos propios de vibración de un recinto rectangular en tres tipos. Los Modos Axiales son aquellos cuya dirección de propagación es paralela a un eje donde la onda estacionaria se produce entre dos planos paralelos, mientras que los Modos Tangenciales son los que presentan sólo dos componentes del vector de onda, siendo el tercero nulo, los mismos que se producen cuando la dirección de propagación de las tres ondas correspondientes permanece paralela al plano de uno de los cerramientos. Por otro lado, los Modos Oblicuos son aquellos que corresponden a los valores no nulos de $n_{x}, n_{y}, n_{z}$. La dirección de propagación de las ondas correspondientes no es paralela a ningún plano de los cerramientos.

En relación a la presión sonora, y en comparativa entre los tres tipos de modos, debemos tener en cuenta que los modos tangenciales tendrán un nivel de presión sonora de $3 \mathrm{~dB}$ inferior al de los modos axiales y los oblicuos $6 \mathrm{~dB}$.

\section{Análisis modal de la Cámara Semianecoica}

Una vez determinadas las medidas de la cámara, se aplicó la Ecuación 5 con los valores mostrados en la Tabla 3, para determinar los diferentes modos de vibración.

Se presenta principal interés en el análisis de los Modos Axiales, pues como se dijo antes, la presión sonora de los modos tangenciales y oblicuos, se encuentran 3 y $6 \mathrm{~dB}$ debajo del NPS de los axiales. La Figura 6 representa los modos propios de la sala en un espectro frecuencial de $0-250 \mathrm{~Hz}$. Como se ve, existe cierta coloración sobre los $200 \mathrm{~Hz}$, que como resultado, se presentarían incrementos frecuenciales por efecto del fenómeno de resonancia.

Tabla 3: Valores requeridos por la Fórmula de Rayleigh

\begin{tabular}{|c|c|c|c|}
\hline$c$ & Largo & Ancho & Alto \\
\hline 144 & 5.97 & 5.60 & 2.83 \\
\hline
\end{tabular}




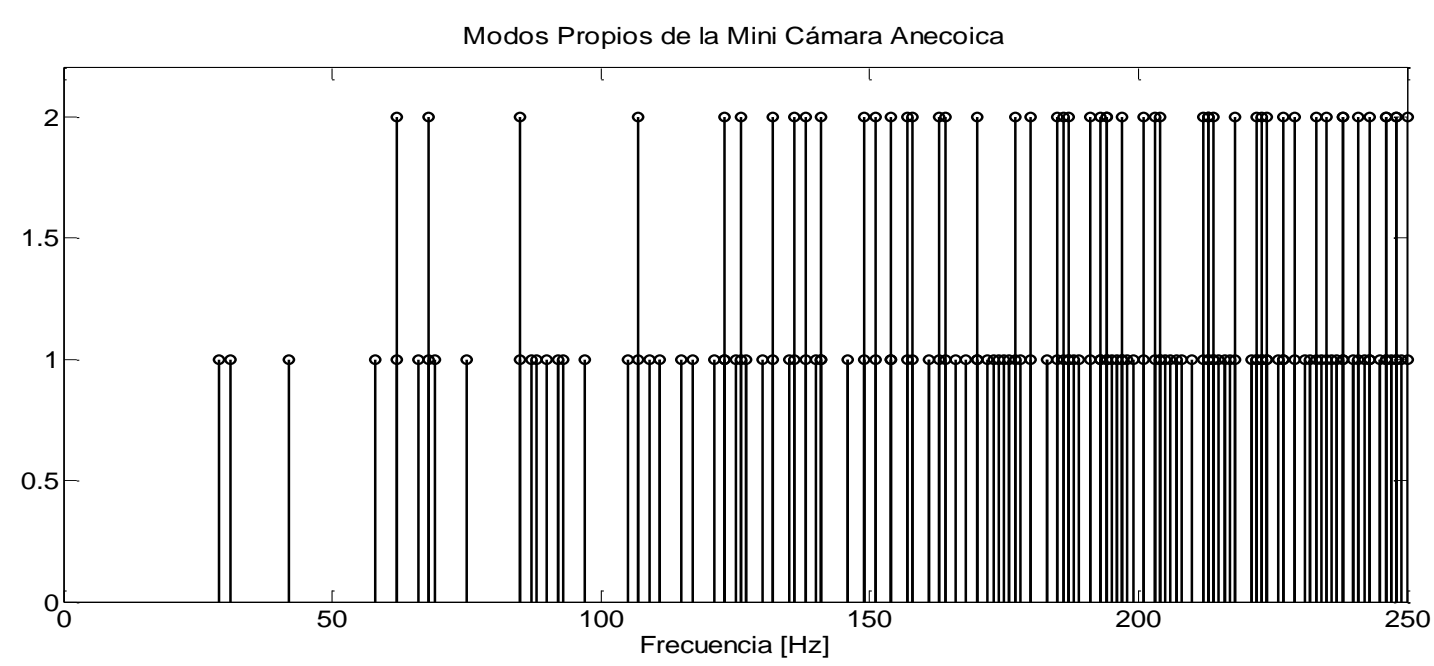

Fig. 6: Modos Propios de la Sala de Pruebas.

\section{ANÁLISIS Y EVAUACIÓN DE LA EFICIENCIA DE LA CÁMARA}

El ruido que se origina en el motor producto de la combustión, se debe a vibraciones producidas por los componentes del mismo, lo que hace que la identificación del origen de una vibración que genere un ruido anormal consecuencia de un fallo de funcionamiento sea compleja. Esta complejidad se incrementa debido a que la estructura general del motor está sometida a diferentes fuerzas de excitación de características diferentes durante el proceso de combustión. Estas fuerzas generan dos tipos de fuentes de ruido en los MCIA, estas fuentes incluyen principalmente al ruido de combustión y al ruido mecánico.

El Ruido de Combustión es producido por las fuerzas generadas por el aumento brusco de la presión en el cilindro, que se origina después del periodo del tiempo de retraso, lo cual provoca un incremento del nivel en la región de alta frecuencia del espectro de la señal, resultando en la vibración estructural del bloque y la excitación de la resonancia del gas en el interior de la cámara de combustión, radiándose de esta manera ruido, el cual se caracteriza por ser la principal fuente de emisión sonora en los Motores Diésel, con una contribución al ruido global de aproximadamente un $80 \%$.

El Ruido Mecánico, es generado por los impactos inducidos por el proceso de combustión sobre la estructura del motor y las partes en movimiento del mismo (Nguyen, 2012). El ruido proveniente de las fuentes mecánicas generalmente domina el rango de altas frecuencias a altas velocidades de funcionamiento. El nivel del ruido mecánico se incrementa de manera más rápida que el del ruido de combustión cuando la velocidad del motor aumenta, así la contribución de ruido mecánico al ruido total llega a ser mayor con el aumento de la carga y la velocidad (Lee et al, 2008).

\section{Modelo de Análisis}

El análisis de la forma de respuesta de la sala y de su eficiencia se realizó a partir de la determinación de la calidad del espectro sonoro muestreado en diferentes condiciones de entorno y funcionamiento de un Motor Diésel Hyundai Santa Fe 2.0 CRDI, cuyas características se muestran en la Tabla 4. Las señales acústicas se obtuvieron con un Medidor del Nivel de Presión Sonora (Sonómetro) presentando las características detalladas en la Tabla 5.

Tabla 4: Características del Motor Diésel Hyundai Santa Fe 2.0 CRDI

\begin{tabular}{|l|c|}
\hline \multicolumn{1}{|c|}{ Descripción } & Especificación \\
\hline Número de Cilindros & 4 \\
\hline Cilindrada & $1991 \mathrm{~cm} 3$ \\
\hline Diámetro del Cilindro & $83 \mathrm{~mm}$ \\
\hline Carrera & $92 \mathrm{~mm}$ \\
\hline Relación de Compresión & $17.7: 1$ \\
\hline Orden de Inyección & $1-3-4-2$ \\
\hline Torque / rpm & $421 \mathrm{~N} . \mathrm{m} / 1800$ \\
\hline Potencia Máxima & $111 \mathrm{KW}$ \\
\hline
\end{tabular}


Las posiciones de medida entorno a la fuente (MCIA) se encuentran esquematizadas en la Figura 7 . Esta distribución es de carácter aleatorio con el fin de determinar nodos recurrentes para el análisis de espectros. Las coordenadas de posicionamiento de estos nodos de medida se encuentran detallados en la Tabla 6. Dentro de las técnicas existentes para el procesamiento de señales, la aplicada en este trabajo es el análisis de señales en el dominio de la frecuencia mediante la Transformada Rápida de Fourier (FFT).

Tabla 5: Características del Sonómetro 3M Modelo 1100

\begin{tabular}{|l|l|}
\hline & \multicolumn{1}{|c|}{ Especificación } \\
\hline Intervalo de Medición & $30-140 \mathrm{dBA}$ \\
\hline Tamaño del Micrófono & $0.52^{\prime \prime}(13.5 \mathrm{~mm})$ \\
\hline Tipo de Micrófono & Eléctrico \\
\hline Filtros Internos & $\mathrm{A}, \mathrm{C}$ \\
\hline Salidas & $\mathrm{AC} / \mathrm{DC}$ \\
\hline Temperatura de Operación & $-10^{\circ} \mathrm{C}$ a $50^{\circ} \mathrm{C}$ \\
\hline
\end{tabular}

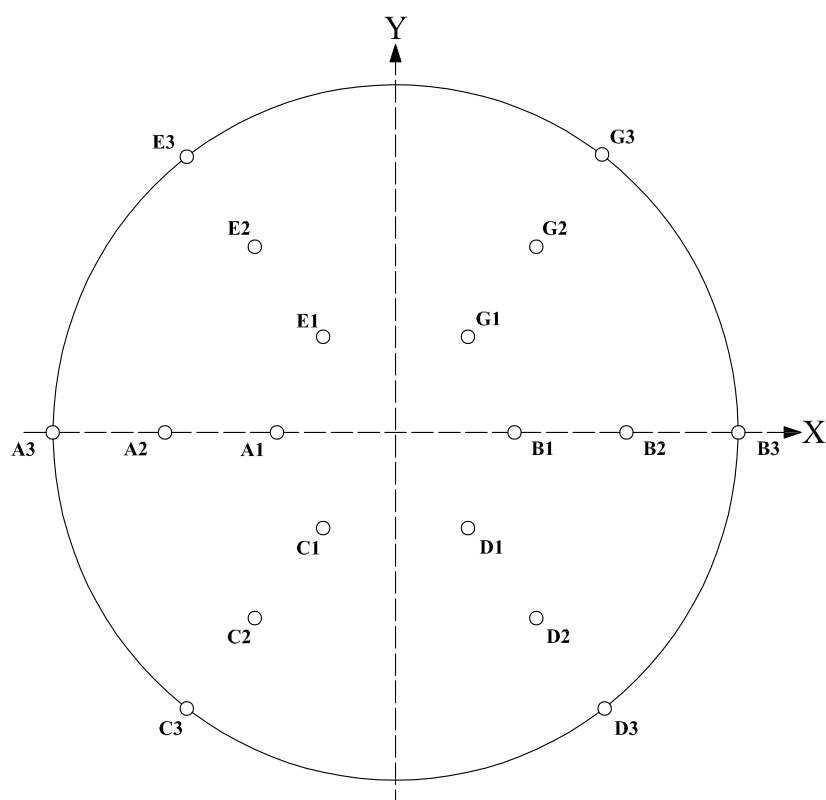

Fig. 7: Posiciones del Sonómetro para la toma de datos

Tabla 6: Coordenadas de Posicionamiento del Sonómetro

\begin{tabular}{|c|c|c|}
\hline Nodo & $x$ & $y$ \\
\hline A1 & $-0,53$ & 0,00 \\
\hline A2 & $-1,03$ & 0,00 \\
\hline A3 & $-1,53$ & 0,00 \\
\hline B1 & 0,53 & 0,00 \\
\hline B2 & 1,03 & 0,00 \\
\hline B3 & 1,53 & 0,00 \\
\hline C1 & $-0,32$ & $-0,42$ \\
\hline C2 & $-0,62$ & $-0,81$ \\
\hline C3 & $-0,93$ & $-1,2$ \\
\hline
\end{tabular}

\begin{tabular}{|c|c|c|}
\hline Nodo & $x$ & $y$ \\
\hline D1 & 0,32 & $-0,42$ \\
\hline D2 & 0,62 & $-0,81$ \\
\hline D3 & 0,93 & $-1,2$ \\
\hline E1 & $-0,32$ & 0,42 \\
\hline E2 & $-0,62$ & 0,81 \\
\hline E3 & $-0,93$ & 1,2 \\
\hline G1 & 0,32 & 0,42 \\
\hline G2 & 0,62 & 0,81 \\
\hline G3 & 0,93 & 1,2 \\
\hline
\end{tabular}

\section{RESULTADOS Y DISCUSIÓN}

Se presenta una selección de los resultados obtenidos en este trabajo. Para determinar la eficiencia de la cámara se realiza un análisis específico para las diferentes RPM de funcionamiento del motor, bajo parámetros de funcionamiento normal, y posteriormente, bajo parámetros de criticidad del mismo, al emular un fallo de inyección en el cilindro №1. Este análisis fue llevado a cabo en dos entornos de medida, el primero con el recinto sin ningún acondicionamiento acústico, mientras que el segundo fue ejecutado en el mismo recinto, acondicionado como Mini Cámara Anecóica. 


\section{Primer Entorno: Sala sin Acondicionamiento}

Bajo el régimen de ralentí (820 rpm), aleatoriamente se presentan los resultados obtenidos. La Figura 8a muestra la firma espectral obtenida a lo largo de los nodos C1, C2, C3, donde se observa la distorsión que presenta el espectro, como resultado de que a bajas frecuencias se excitan los modos propios de vibración de la sala, entrando en una etapa de resonancia que dificulta una correcta interpretación del funcionamiento del motor.

Para el análisis del régimen de $1500 \mathrm{rpm}$, en la Figura 8b se observa el resultado correspondiente a los nodos D1, D2, D3, donde con menor amplitud aparece el armónico fundamental correspondiente a la frecuencia de encendido. Obsérvese como el segundo armónico presenta una amplitud mayor enmascarando los armónicos fundamentales por la respuesta vibrante de la sala. Por su parte la Figura 8c detalla el análisis espectral aleatorio correspondiente a un régimen de $2000 \mathrm{rpm}$, donde se observa la evolución de los espectros en relación a la distancia de la toma de datos, lo cual no representa un patrón característico, sino más bien no guarda relación alguna entre sus muestras. Se observa que en el nodo G2, el segundo armónico tiene mayor amplitud que el primero, correspondiente a la frecuencia de encendido.
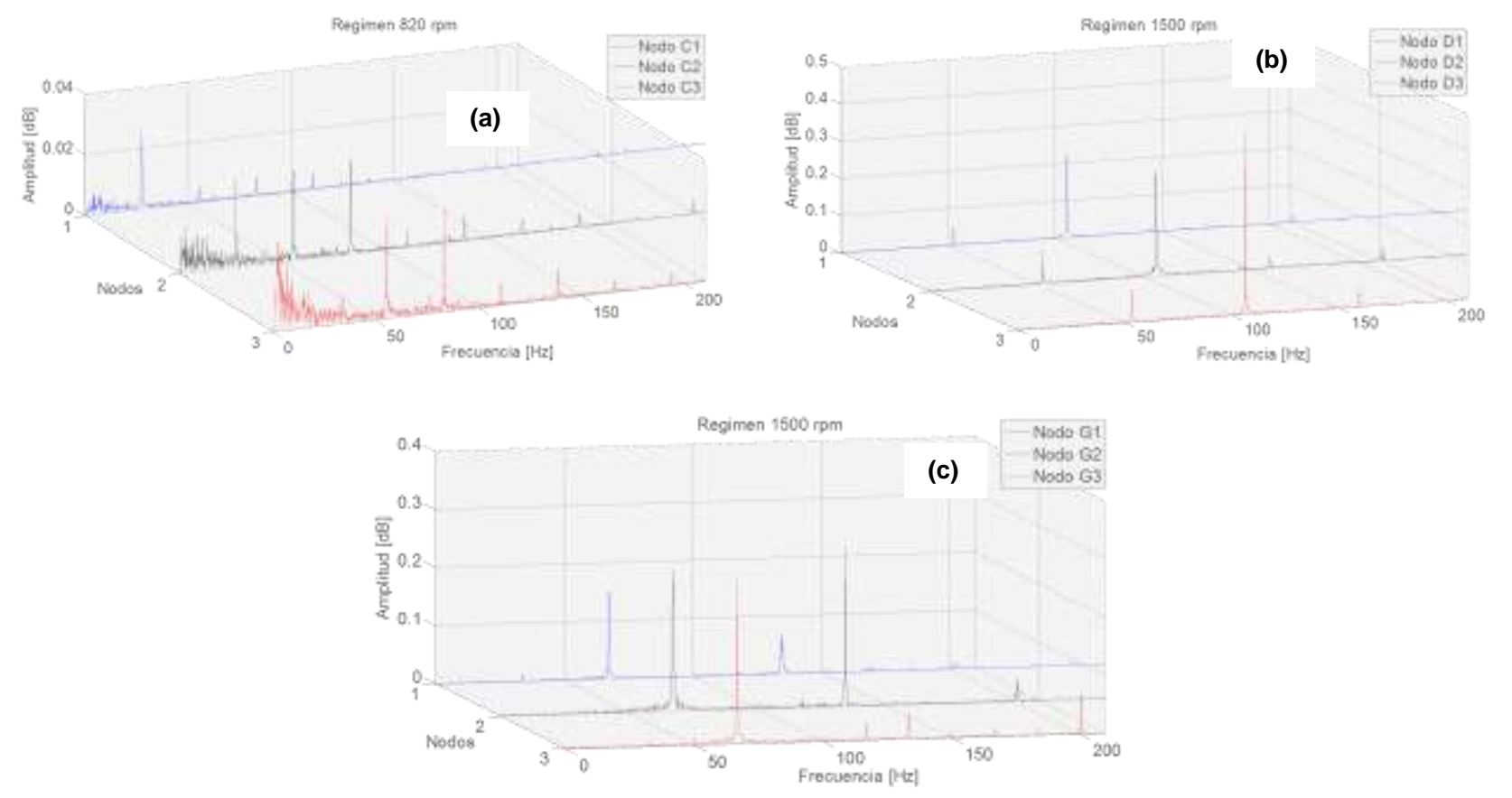

Fig. 8: Análisis espectral de los Nodos: (a) C1,C2,C3 a 820 rpm; (b) D1,D2, D3 a 1500 rpm; (c) G1,G2,G3 a 2000 rpm

\section{Segundo Entorno: Sala con Acondicionamiento (Cámara Semianecoica)}

Como se ha visto anteriormente no existe un patrón estable que permita una correcta aplicación del Análisis de Ruido como técnica de mantenimiento predictivo, debido a que las señales emitidas por la fuente entran en contacto con los modos propios de vibración de la sala, por lo que obliga a atenuar la presencia de estos modos, mejorando la acústica del recinto a través del acondicionamiento acústico, a fin de garantizar un entorno de medida fiable. La Figura 9 nos muestra las firmas espectrales después del acondicionamiento acústico / anecoico. Como se observa, se han obtenido los picos característicos correspondientes a la frecuencia de encendido $(1 x)$ y sus correspondientes sub-armónicos $(2 x, 3 x)$. Considerando que el régimen de giro es $2000 \mathrm{rpm}$ y que el motor es un 4 cilindros de 4 tiempos, es decir para cada ciclo el cigüeñal gira dos veces, se obtienen los valores frecuenciales de éstos picos, los mismos que se encuentran detallados en la Tabla 7.

Tabla 7: Máximos característicos del Motor a un régimen de 2000 rpm

\begin{tabular}{|c|c|c|c|}
\hline Nodo & $\begin{array}{c}\text { 1er Armónico } \\
(1 x)\end{array}$ & $\begin{array}{c}\text { 2do Armónico } \\
(2 x)\end{array}$ & $\begin{array}{c}\text { 3er Armónico } \\
\text { (3x) }\end{array}$ \\
\hline G1 & $68.6 \mathrm{~Hz}$ & $137.2 \mathrm{~Hz}$ & $205,8 \mathrm{~Hz}$ \\
\hline G2 & $68,6 \mathrm{~Hz}$ & $237,2 \mathrm{~Hz}$ & $205,8 \mathrm{~Hz}$ \\
\hline G3 & $68,2 \mathrm{~Hz}$ & $136,2 \mathrm{~Hz}$ & $204,8 \mathrm{~Hz}$ \\
\hline
\end{tabular}



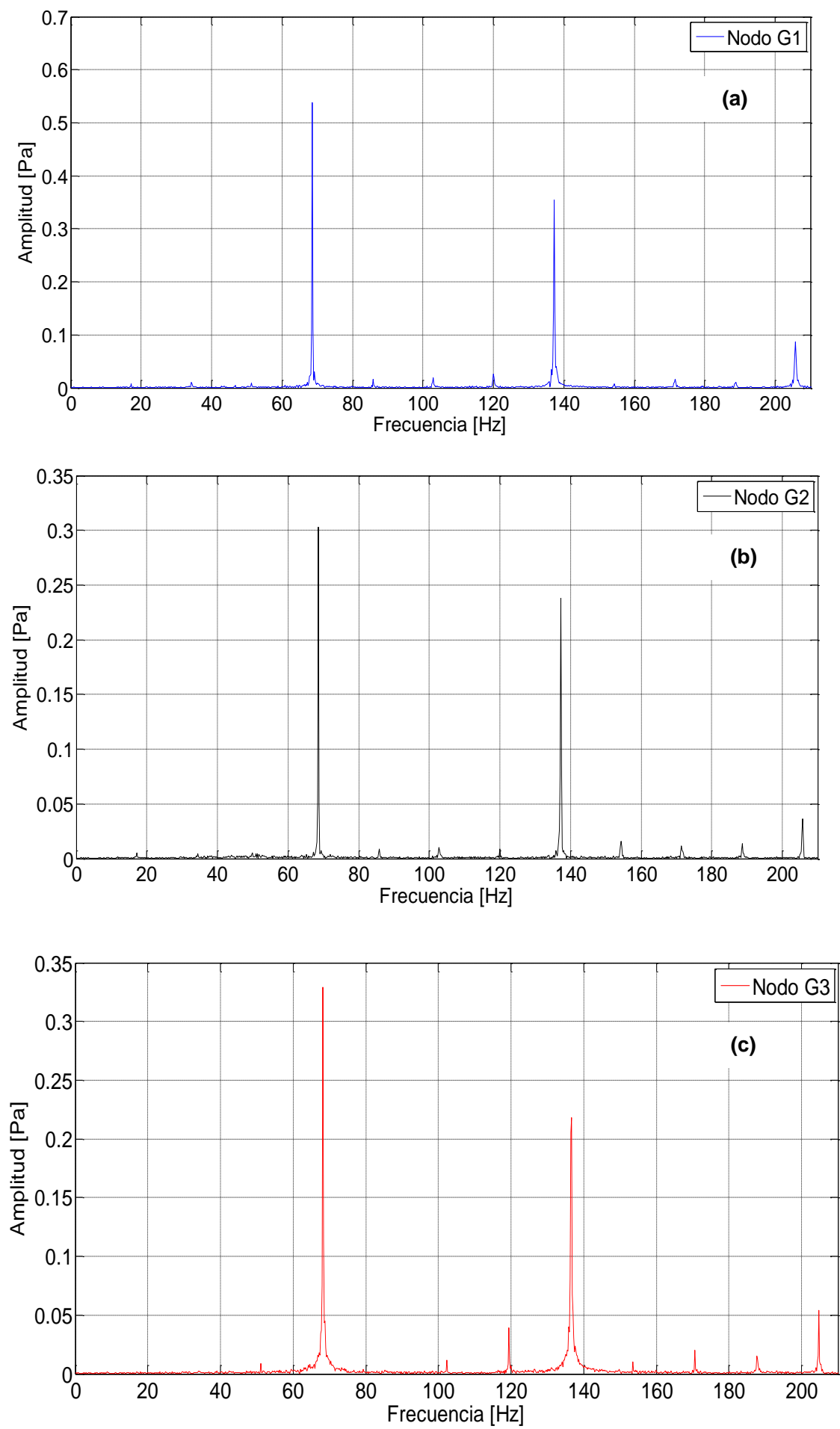

Fig. 9: Análisis espectral de los Nodos G1, G2, G3 a un régimen de 2000 rpm.

El análisis realizado en la Cámara Semianecoica permitió establecer patrones de medida con espectros frecuenciales limpios que den paso al Análisis de Ruido como técnica aplicable al diagnóstico de Motores, dentro del marco establecido por el Mantenimiento Basado en Condición conocido como Mantenimiento Predictivo.

En la Figura 10, podemos apreciar un espectro característico cuando el motor se encuentra girando a 2000 rpm con un fallo provocado en el inyector del cilindro 1. Funcionando solamente con tres de sus cuatro cilindros, se observa el pico fundamental de la frecuencia de encendido ( $1 x \mathrm{fe})$, y sus sub-armónicos, con un incremento considerable en la amplitud de su segundo armónico $(2 x \mathrm{fe})$. En este tipo de daños, claramente aparece una frecuencia equivalente a $1 / 4 f e$, con sus respectivos sub-armónicos, y será éste el valor de las bandas laterales que aparezcan junto los armónicos fundamentales (1x fe; $2 x$ fe; $3 x f e$ ). Este valor de $1 / 4$ de la frecuencia de encendido fe aparece a razón del número de cilindros de la fuente en estudio. 


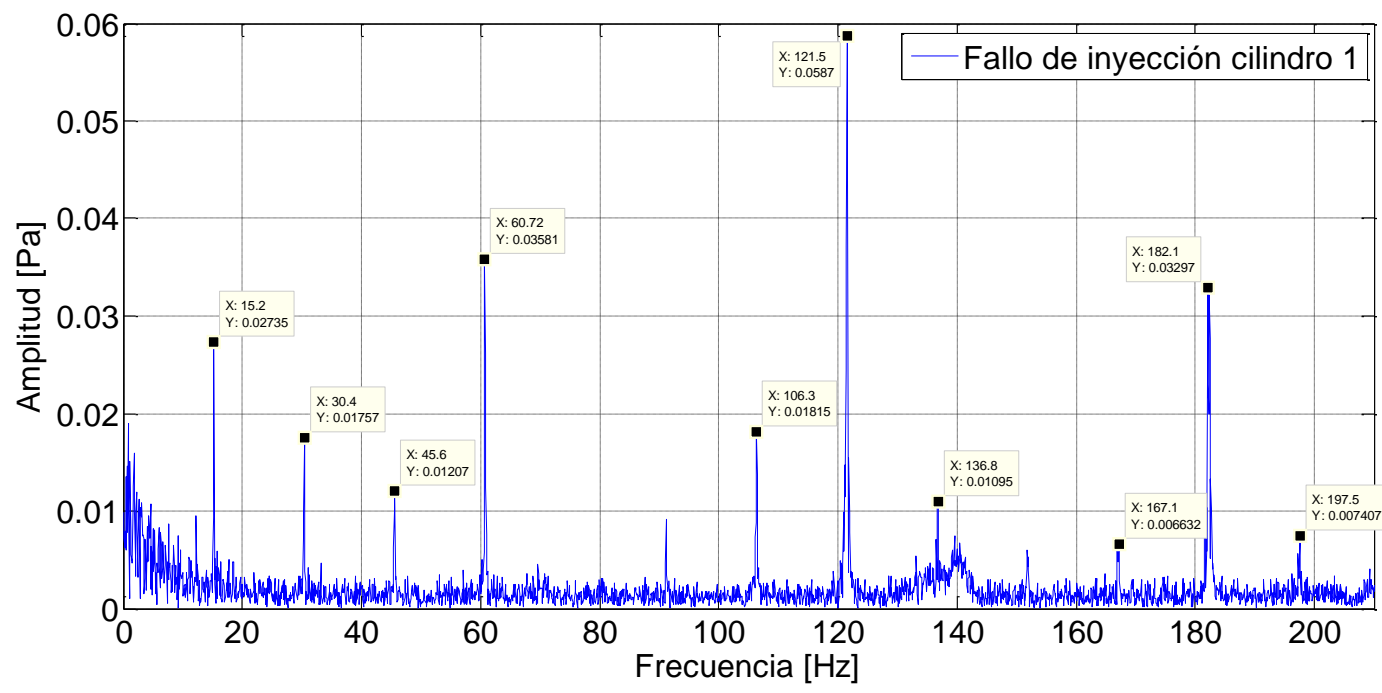

Fig. 10: Análisis espectral en el Nodo G1 a un régimen de 2000 rpm con un fallo provocado

\section{CONCLUSIONES}

El método de Máximos y Mínimos en el tubo de Kundt es un método confiable, el cual se aplicó para el análisis de diferentes materiales, obteniendo datos fiables del coeficiente de absorción y pérdida por transmisión del ruido, consiguiendo un adecuado acondicionamiento acústico de la cámara semianecoica.

En específico se busca: "conseguir aislar completamente el ruido exterior y obtener únicamente el ruido presente en los mecanismos analizados de manera muy puntual." razón por la cual, el trabajo se orientó al diseño y construcción de una cámara semianecoica a partir de los armónicos fundamentales de la frecuencia de encendido $1 x$ de un Motor de Combustión Interna como resultado del procesamiento y análisis de las señales acústicas obtenidas para la determinación de la frecuencia de corte, es decir un entorno que elimina el eco y la reverberación que pueda captar el micrófono. Se obtuvo un ruido de fondo menor a los 25 dB en ponderación "A" y un promedio general del coeficiente de absorción en las bandas de octava del 90\% con un rango de frecuencias desde aproximadamente los $125 \mathrm{~Hz}$ a los $4 \mathrm{kHz}$, y una frecuencia de corte de $200 \mathrm{~Hz}$, lo cual permite una correcta aplicación de la Técnica de Análisis de Ruido, como una herramienta de Mantenimiento Predictivo, que permita monitorizar la evaluación de parámetros específicos del funcionamiento del motor.

Se ha tomado como ejemplo la simulación del fallo de inyección, pudiendo ampliarse a muchos fallos más a partir de la obtención, como punto de partida, las firmas espectrales claras y específicas referentes al correcto funcionamiento del motor y establecer un patrón de firmas correspondientes a fallos característicos y comunes en el motor, como la variación de cargas, sensores y potencia.

\section{REFERENCIAS}

Ambardar, A., Procesamiento de Señales Analógicas y Digitales, $2^{\text {a }}$ edición, 248-284, Editorial Thomson, México D.F (2002)

Davidson, M., Hemi-anechoic Chamber Qualification and Comparison of Room Qualification Standards, doi: 10.1121/1.4877859, The Journal of the Acoustical Society of America, 135(4), 2379 (2014)

Kim, D., D. Kim y D. Choi, Design of a Super Wide-band EM Wave Absorber for a General Purpose Anechoic Chamber, doi:10.1016/j.cap.2006.09.033, Current Applied Physics, Elsevier, 7(5), 457-462 (2007)

Lee M., J. Bolton y S. Shu, Estimation of the Combustion-related Noise Transfer Matrix of a Multi-cylinder Diesel Engine, doi: 10.1088/0957-0233/20/1/015106, Measurement Science And Technology 20(1):015106 . (2008)

Martyr, A. y M. Plint, Chapter 18 - Anechoic Test Cells: NVH and EMC Testing, in Engine Testing, Editorial Elsevier, Fourth Edition, pp. 483-500, Oxford, UK (2012) 
Möser, M. y J. Barros, Absorción del Sonido, en Ingeniería Acústica, Editorial Springer, pp.167-212, Berlin, Germany (2009)

Nguyen, T, K. Yuichiro y M. Masato, Study on Combustion Noise from a Running Diesel Engine Based on Transient Combustion Noise Generation Model, http://tech.jsae.or.jp/ijae/detail.aspx?pno=20124039, ISSN 2185-0992, International Journal of Automotive Engineering, Vol.3, No.4, pp.131-140 (2012)

Piedrahita, Y. y , F. Fajardo, Construcción de una Cámara Anecoica para la Caracterización de la Pérdida de Transmisión Sonora, http://www.scielo.br/scielo.php?script=sci_arttext\&pid=S1806-

1172012000400005\&lang=pt,ISSN 1806-1117, Revista Brasileira de Ensino de Física, 34(4), 1-9 (2012)

Rodríguez, G. y A. Escamilla, Rediseño de las Cámaras Anecoica y Reverberante (Ecoica) de la ESIME Zacateco, Tesis de Titulación, Escuela Superior de Ingeniería Mecánica y Eléctrica, Instituto Politécnico Nacional, México, (2011)

Schneider, A., Numerical Prediction of the Quality of an Anechoic Chamber in the Low Frequency Range, doi:10.1016/j.jsv.2008.08.019, Journal of Sound and Vibration, Elsevier, 320(4-5), 990-1003 (2009)

Strutt, J., Chapter XIII - Aerial Vibrations in a Rectangular Chamber, in The Theory of Sound, 2nd Edition, Cambridge University Press, Vol. 2, pp 65-84, Cambridge, UK (2011) 